\title{
Comprehensive interrogation of natural TALE DNA-binding modules and transcriptional repressor domains
}

\author{
Le Cong ${ }^{1,2,3}$, Ruhong Zhou ${ }^{4}$, Yu-chi Kuo ${ }^{1,2}$, Margaret Cunniff',2 \& Feng Zhang ${ }^{1,2}$
}

Transcription activator-like effectors are sequence-specific DNA-binding proteins that harbour modular, repetitive DNA-binding domains. Transcription activator-like effectors have enabled the creation of customizable designer transcriptional factors and sequence-specific nucleases for genome engineering. Here we report two improvements of the transcription activator-like effector toolbox for achieving efficient activation and repression of endogenous gene expression in mammalian cells. We show that the naturally occurring repeat-variable diresidue Asn-His $(\mathrm{NH})$ has high biological activity and specificity for guanine, a highly prevalent base in mammalian genomes. We also report an effective transcription activator-like effector transcriptional repressor architecture for targeted inhibition of transcription in mammalian cells. These findings will improve the precision and effectiveness of genome engineering that can be achieved using transcription activator-like effectors.

\footnotetext{
${ }^{1}$ Broad Institute of MIT and Harvard, Cambridge, Massachusetts 02142, USA. ${ }^{2}$ McGovern Institute for Brain Research, MIT, Department of Brain and Cognitive Sciences, Massachusetts Institute of Technology, Cambridge, Massachusetts 02142, USA. ${ }^{3}$ Program in Biological and Biomedical Sciences, Harvard Medical School, Boston, Massachusetts 02115, USA. ${ }^{4}$ Computational Biology Center, IBM Thomas J. Watson Research Center, Yorktown Heights, New York 10598, USA. Correspondence and requests for materials should be addressed to F.Z. (email: zhang_f@mit.edu).
} 
T ranscription activator-like effectors (TALEs) are bacterial effector proteins found in Xanthamonas sp. and Ralstonia $s p$. Each TALE contains a DNA-binding domain consisting of 34 amino-acid tandem repeat modules, where the twelfth and thirteenth residues of each module, referred to as repeat-variable diresidues (RVDs), specify the target DNA base ${ }^{1,2}$. Four of the most abundant RVDs from naturally occurring TALEs have established a simple code for DNA recognition (for example, NI for adenine, $\mathrm{HD}$ for cytosine, NG for thymine, and NN for guanine or adenine) $)^{1,2}$. Using this simple code, TALEs have been developed into a versatile platform for achieving precise genomic and transcriptomic perturbations across a diverse range of biological systems $s^{3-8}$. However, two limitations remain: first, an RVD capable of robustly and specifically recognizing the DNA base guanine-a highly prevalent base in mammalian genomes ${ }^{9}$ - is lacking; second, a viable TALE transcriptional repressor for mammalian applications has remained elusive, but would be highly desirable for a variety of synthetic-biology and disease-modelling applications ${ }^{9}$.

To address these two limitations, we conducted a series of screens and found that of all naturally occurring TALE RVDs, the previously unidentified RVD Asn-His (NH) can be used to achieve guaninespecific recognition. Furthermore, we show that the $\mathrm{mSin}$ interaction domain (SID) ${ }^{10}$ can be fused to TALEs to facilitate targeted transcriptional repression of endogenous mammalian gene expression. These advances further improve the power and precision of TALE-based genome engineering technologies, enabling efficient bimodal control of mammalian transcriptional processes.

\section{Results}

Screening of novel TALE RVDs. Previously, the RVD NK was reported to have more specificity for guanine than $\mathrm{NN}^{4}$. However, recent studies have shown that substitution of NK with NN leads to substantially lower levels of activity ${ }^{11}$. To identify a more specific guanine-binding RVD with higher biological activity, we identified and evaluated a total of 23 naturally occurring RVDs (Fig. 1) from the set of known Xanthomonas TALE sequences in Genbank. In order to directly compare the DNA-binding specificity and activity of all RVDs in an unbiased manner, we designed a set of 23 12.5-repeat TALEs where we systematically substituted RVDs 5 and 6 with the 23 naturally occurring RVDs (RVD-TALEs; Fig. 1a). This design allowed us to maintain a consistent RVD context surrounding the two varied RVD positions. In addition, we fused a Gaussian luciferase gene (Gluc) with a 2A peptide linker to the RVDTALEs to control for the differences in TALE protein expression levels (Fig. 1a). We used each RVD-TALE (for example, NI-TALE, HDTALE, and so on) to assess the base preference and activity strength of its corresponding RVD-this is measured by comparing each RVD-TALE's ability to activate transcription from each of the four base-specific Cypridina luciferase reporter (Cluc) plasmids with A, G, T and C substituted in the sixth and seventh positions of the TALE-binding site (A-, G-, T- or C-reporters; Fig. 1a).

The 23 RVD-TALEs exhibited a wide range of DNA base preferences and biological activities in our reporter assay (Fig. 1b). In particular, NH- and HN-TALEs activated the guanine reporter preferentially and at similar levels as the NN-TALE. Interestingly, the NH-TALE also exhibited significantly higher specificity for the G-reporter than the NN-TALE (ratio of G- to A-reporter activations: 16.9 for NH-TALE and 2.7 for NN-TALE; Fig. 1b), suggesting that NH might be a better RVD for targeting guanines.

Our computational analysis of TALE-RVD specificity using a recently published crystal structure of TALE-DNA complex ${ }^{12}$ also suggests that $\mathrm{NH}$ has a significantly higher affinity for guanine than NN (Fig. 2). We found that substitution of $\mathrm{NN}$ with $\mathrm{NH}$ in one repeat within the TALE DNA-binding domain resulted in a gain
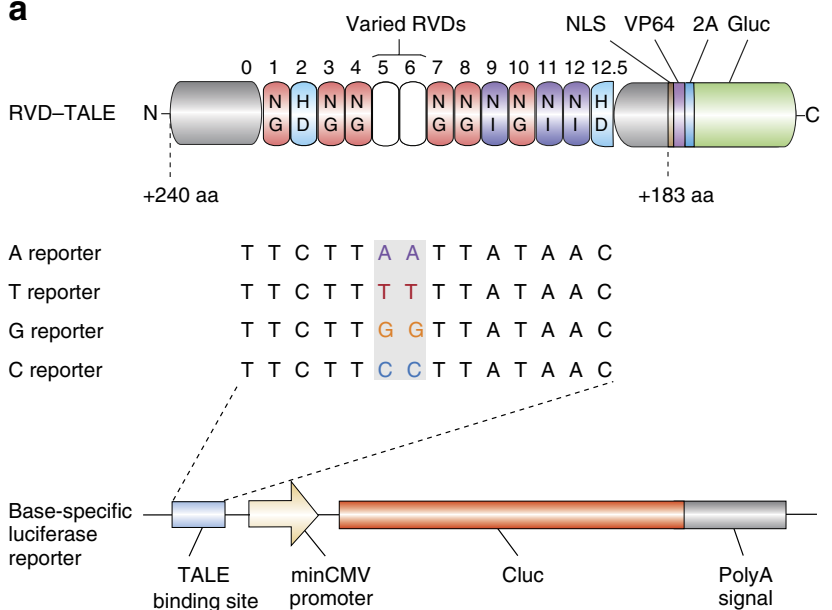

b

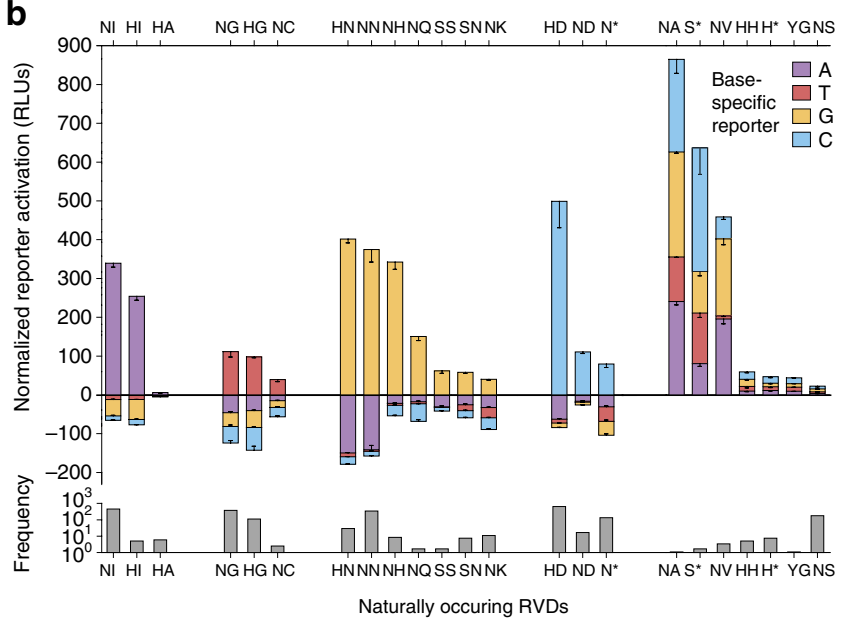

Figure 1 | Identification of an optimal guanine-specific RVD. (a) Design of the TALE RVD screening system. Each RVD-screening TALE (RVD-TALE) contains 12.5 repeats with RVDs 5 and 6 substituted with the 23 naturally occurring RVDs, and is fused to a Gaussia luciferase gene via a 2A peptide linker. The truncations used for the TALE is marked at the amino and carboxy termini with numbers of amino acids (aa) retained (top). Four different base-specific reporters with $A, T, G$ and $C$ substituted in the sixth and seventh nucleotides of the binding site are used to determine the base specificity of each RVD (middle). Each reporter is constructed by placing the TALE-binding site upstream of a minimal CMV promoter driving Cypridina luciferase (bottom). (b) Base preference of each natural RVD (top) is determined by measuring the levels of relative luminescence unit (RLU) for each basespecific reporter after background subtraction and normalization based on TALE protein expression level (top). We clustered RVDs according to their base preference after performing one-way ANOVA tests on each RVD. For RVDs with a single statistically significant reporter activity $(P<0.05$, one-way ANOVA), we plotted the reporter activity of the preferred base above the $x$ axis, whereas the reporter activities for the non-preferred bases are shown below the $x$ axis as negative. We clustered and ranked the RVDs without a single preferred base according to their total activity level. The abundance of each RVD in natural TALE sequences, as determined using all available Xanthomonas TALE sequences in GenBank, is plotted on a log scale (bottom). All bases in the TALE-binding site are color-coded (purple for A, red for T, orange for G and blue for C). NLS, nuclear localization signal; VP64, VP64 viral activation domain; 2A, 2A peptide linker; Gluc, Gaussia luciferase gene; minCMV, minimal CMV promoter; Cluc, Cypridina luciferase gene; polyA signal, poly-adenylation signal. All results are collected from three independent experiments in HEK 293FT cells. Error bars indicate s.e.m.; $n=3$. 


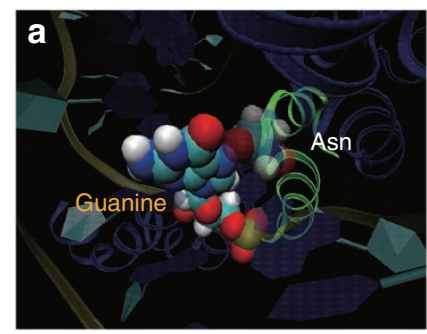

$\mathrm{RVD}=\mathrm{NN}$ (reference)

$\Delta \Delta \mathrm{G}=0 \mathrm{kcal} \mathrm{mol}^{-1}$

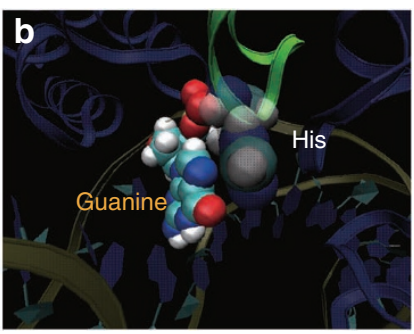

RVD $=\mathrm{NH}$ $\Delta \Delta \mathrm{G}=-0.86 \pm 0.67 \mathrm{kcal} \mathrm{mol}^{-1}$
Figure 2 | Computational analysis of TALE RVD specificity. We

performed extensive free FEP calculations for the relative binding affinities between the TALE and its bound DNA. Images show the three-dimensional configuration and results of the free-energy calculation for NN:G (a) and $\mathrm{NH}: \mathrm{G}$ (b) interactions from one repeat in the TALE-DNA complex. The second amino acid (aa) of the guanine-recognizing RVD (that is, asparagine for RVD NN and histidine for RVD NH) and the guanine base of the bound double-stranded DNA are presented in space filling model and labelled. The free-energy calculation results are listed below their corresponding structures.

of $0.86 \pm 0.67 \mathrm{kcal} \mathrm{mol}^{-1}$ in free energy $(\Delta \Delta G)$ in the DNA-bound state (Fig. 2).

Relative activity and specificity of guanine-binding RVDs. To determine whether $\mathrm{NH}$ and $\mathrm{HN}$ are suitable replacements for $\mathrm{NN}$ as the G-specific RVD, we directly compared the specificity and activity strength of NN, NK, NH and HN. We chose two 18-base-pair (bp) targets within the CACNA1C locus in the human genome and constructed four TALEs for each target, using NN, NK, NH or HN as the G-targeting RVD (Fig. 3a). As the screening result (Fig. 1b) suggested that $\mathrm{HN}$ might be less discriminatory than $\mathrm{NH}$ when the targeted base is A instead of G, we first designed a luciferase assay to further characterize the G-specificity of each RVD. For each $C A C$ $N A 1 C$ target site, we constructed four luciferase reporters: wildtype genomic target, and wild-type target with 2, 4 or all guanines mutated into adenines (Fig. 3a, G-to-A reporters), and compared the activity of each TALE using these reporters (Fig. 3a). For both CACNA1C target sites, we found that the TALE with $\mathrm{NH}$ as the G-targeting RVD exhibited significantly higher specificity for guanine over adenine than the corresponding NK-, HN- and NNcontaining TALEs. For target site 1, introduction of two $\mathrm{G}$ to A mutations led to $35.4 \%$ (TALE1-NN), $40.3 \%$ (TALE1-NK), 71.4\% (TALE1-NH) and $30.8 \%$ (TALE1-HN) of reduction in luciferase activity. For target site 2, two G-to-A mutations led to $21.8 \%$ (TALE2NN), 36.3\% (TALE2-NK), 66.1\% (TALE2-NH) and 13.9\% (TALE2$\mathrm{HN}$ ) reduction in reporter activity. Additional G-to-A mutations resulted in further reduction of reporter activity, with $\mathrm{NH}$ exhibiting the highest level of discrimination (Fig. 3a). NH TALEs also exhibited significantly higher levels of reporter induction than NK TALEs (1.9 times for site 1 and 2.7 times for site 2), and comparable to NN and NH TALEs (Fig. 3a). Thus, we decided to focus on the RVDs NN, NK, NH and HN in subsequent experiments to assess their usefulness in modulating transcription at endogenous human genome targets.

Evaluation of guanine-binding RVDs at endogenous genome loci. Using quantitative reverse transcriptase PCR, we further compared the performance of $\mathrm{NN}, \mathrm{NK}, \mathrm{NH}$ and $\mathrm{HN}$ for targeting endogenous genomic sequences. We tested the ability of NN, NK, NH and $\mathrm{HN}$ TALEs to activate CACNA1C transcription by targeting the two endogenous target sites (Fig. 3b). To control for differences in TALE expression levels, all TALEs were fused to 2A-GFP and exhibited similar levels of green fluorescent protein (GFP) fluorescence ${ }^{3}$. The endogenous activity of each TALE corresponded to the reporter assay. Both TALE1-NH and TALE2-NH were able to achieve similar levels of transcriptional activation as TALE1-NN and TALE2-NN ( $\sim 5$ fold and $\sim 3$ fold activation for targets 1 and 2 , respectively) and twice more than TALE1-NK and TALE2-NK (Fig. 3b). Although TALE1-HN and TALE2-HN exhibited comparable activity with TALEs bearing RVDs NN and NH, the lack of specificity in distinguishing guanine and adenosine bases as shown in previous test (Fig. 3a) does not warrant the superiority of HN over existing guanine-binding RVDs. On the other hand, RVD NH exhibited similar levels of activity as NN and with higher specificity for guanine.

Development of mammalian TALE transcriptional repressors. Having identified $\mathrm{NH}$ as a more-specific G-recognizing RVD, we sought to develop a mammalian TALE-repressor architecture to enable researchers to suppress transcription of endogenous genes. TALE repressors have the potential to suppress the expression of genes as well as non-coding transcripts such as microRNAs, rendering it a highly desirable tool for testing the causal role of specific genetic elements. In order to identify a suitable repression domain for use with TALEs in mammalian cells, we used a TALE targeting the promoter of the human SOX2 gene to evaluate the transcriptional repression activity of a collection of candidate repression domains (Fig. 4a). We selected repression domains across a range of eukaryotic host species to increase the change of finding a potent synthetic repressor, including the PIE-1 repression domain (PIE-1) ${ }^{13}$ from Caenorhabditis elegans, the QA domain within the Ubx gene (Ubx-QA) ${ }^{14}$ from Drosophila melanogaster, the IAA28 repression domain (IAA28-RD) ${ }^{15}$ from Arabidopsis thaliana, the SID ${ }^{10}$, Tbx3 repression domain (Tbx3-RD) and the Krüppel-associated box (KRAB) ${ }^{16}$ repression domain from Homo sapiens (Fig. 4b). As different truncations of KRAB have been known to exhibit varying levels of transcriptional repression ${ }^{16}$, we tested three different truncations of KRAB (Fig. 4c). We expressed these candidate TALE repressors in HEK 293FT cells and found that TALEs carrying two widely used mammalian transcriptional repression domains, the $\mathrm{SID}^{10}$ and $\mathrm{KRAB}^{16}$ domains, were able to repress endogenous SOX2 expression, while the other domains had little effect on transcriptional activity (Fig. 4c). To control for potential perturbation of SOX2 transcription owing to TALE binding, expression of the SOX2targeting TALE DNA-binding domain alone without any effector domain had no effect (similar to mock or expression of GFP) on the transcriptional activity of SOX2 (Fig. 4c, null condition). As the SID domain was able to achieve $26 \%$ more transcriptional repression of the endogenous SOX2 locus than the KRAB domain (Fig. 4c), we decided to use the SID domain for our subsequent studies.

To further test the effectiveness of the SID repressor domain for downregulating endogenous transcription, we combined SID with CACNA1C-target TALEs from the previous experiment (Figs 3 and $4 d$ ). Using quantitative reverse transcriptase PCR, we found that replacement of the VP64 domain on CACNA1C-targeting TALEs with SID was able to repress CACNA1C transcription. In addition, similar to the transcriptional activation study (Fig. 3b, left), $\mathrm{NH}$-containing TALE repressor was able to achieve a similar level of transcriptional repression as the NN-containing TALE $(\sim 4$ fold repression), while the TALE repressor using NK was significantly less active ( 2 fold repression, Fig. $4 \mathrm{~d})$. These data demonstrate that SID is indeed a suitable repression domain, while also further supporting $\mathrm{NH}$ as a more suitable G-targeting RVD than NK.

\section{Discussion}

TALEs can be easily customized to recognize specific sequences on the endogenous genome, and can be used to target a diverse range of effector domains to specific genomic loci. Here, we conducted 
a

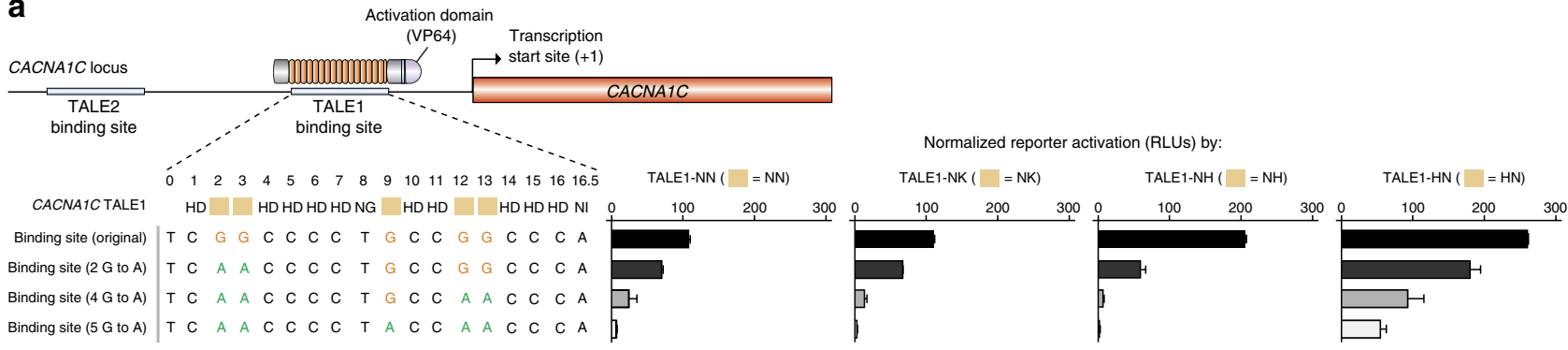

\begin{tabular}{l|llllllllllllllllll} 
Binding site (4 $G$ to $A)$ & T & $C$ & $A$ & $A$ & $C$ & $C$ & $C$ & $C$ & $T$ & $G$ & $C$ & $C$ & $A$ & $A$ & $C$ & $C$ & $C$ & $A$ \\
Binding site $(5 G$ to $A)$ & T & $C$ & $A$ & $A$ & $C$ & $C$ & $C$ & $C$ & $T$ & $A$ & $C$ & $C$ & $A$ & $A$ & $C$ & $C$ & $C$ & $A$
\end{tabular}

$\begin{array}{lllllllllllllllllll}0 & 1 & 2 & 3 & 4 & 5 & 6 & 7 & 8 & 9 & 10 & 11 & 12 & 13 & 14 & 15 & 16 & 16.5\end{array}$ TALE2-NN ( $\left.=\mathrm{NN}\right)$ \begin{tabular}{l|l|llll} 
CACNA1CTALE2 & NG NI NI HD HD NG NGNI & HDNGNI & 0 & 100 & 200 \\
\hline
\end{tabular}

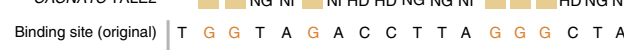

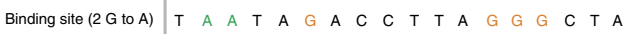
Binding site (4G to $A$ ) Binding site $(6 G$ to $A$ )

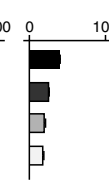

TALE2-NK ( $=$ NK) 200
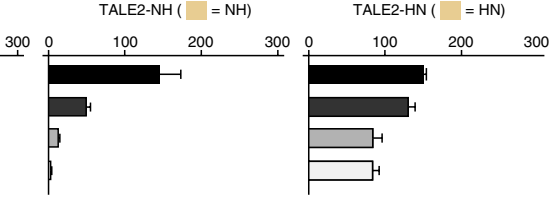

b

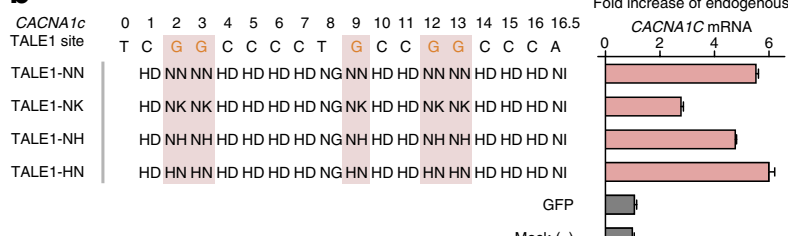

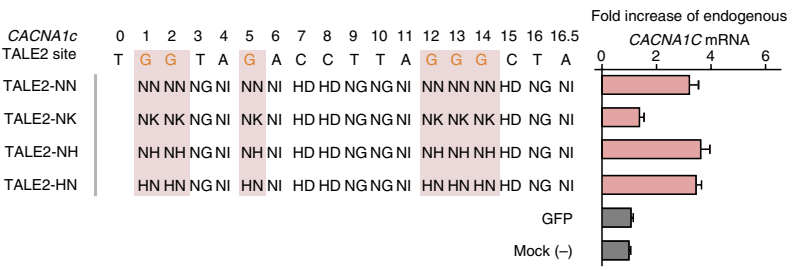

Figure 3 | Characterization of guanine-specific RVDs. (a) Specificity and activity of different guanine-targeting RVDs. Schematic showing the selection of two TALE-binding sites within the CACNATC locus of the human genome. The TALE RVDs are shown above the binding site sequences and yellow rectangles indicate positions of G-targeting RVDs (left). Four different TALEs using NN, NK, NH and HN as the putative G-targeting RVD were synthesized for each target site. The specificity for each putative G-targeting RVD is assessed using luciferase reporter assay, by measuring the levels of reporter activation of the wild-type TALE-binding site and mutant binding sites, with either 2, 4 or all guanines substituted by adenine. The mutated guanines and adenines are highlighted with orange and green, respectively. (b) Endogenous transcriptional modulation using TALEs containing putative G-specific RVDs. TALEs using NN, NK, NH and HN as the G-targeting RVD were synthesized to target two distinct 18-bp target sites in the human CACNA1C locus. Changes in messenger RNA (mRNA) are measured using qRT-PCR as described previously ${ }^{3}$. VP64, VP64 transcription activation domain. All results are collected from three independent experiments in HEK 293FT cells. Error bars indicate s.e.m.; $n=3$.

a series of screens to improve the specificity TALE-DNA recognition as well as expand the functionality of TALEs for modulating endogenous gene transcription.

Our TALE RVD screening results identified a set of novel RVDs with useful activity and specificity. Among them, the RVD NH demonstrated increased specificity for recognizing guanine. While NK was previously shown to be able to target guanine specifically ${ }^{17}$, our endogenous gene modulation tests as well as a recent TALE nuclease study ${ }^{11}$ have shown that TALEs employing NK to target guanine has less than half of the activity compared with TALEs using NN or NH. Our computational modelling result using the recently resolved crystal structure of a TALE-DNA complex ${ }^{12}$ show that the imidazole ring on the histidine residue of the NH RVD has a more compact base-stacking interaction with the target guanine base (Fig. 2), indicating that $\mathrm{NH}$ would be able to bind guanine more tightly than $\mathrm{NN}$ with a $0.86 \pm 0.67 \mathrm{kcal} / \mathrm{mol}$ higher binding affinity and thus suggesting a possible mechanism for its increased specificity for guanine. Therefore based on all of these results from specificity and endogenous activity tests, the RVD NH seems to be a more suitable substitute for NN than NK when higher targeting specificity is desired. Further testing using additional endogenous genomic targets will help validate the broad utility of $\mathrm{NH}$ as a highly specific G-targeting RVD. In addition, the RVD NA exhibited similar levels of reporter activation for all four bases and may be a promising candidate for high efficiency targeting of degenerate DNA sequences in scenarios where non-specific binding is desired ${ }^{18}$.

In addition, our screen of six different repressor domains across a range of host species resulted in the identification of two repressor domains capable of repressing mammalian transcription. The SID domain achieved the strongest level of repression at endogenous mammalian genomic loci. Having the ability to use TALEs to target transcriptional effectors may enable persistent epigenetic modification in the chromatin state of target loci in the endogenous genome. However, future studies will need to elucidate the mechanism of targeted transcriptional modulation by TALE transcription factors and their specificity. The identification of a more stringent G-specific RVD with uncompromised activity strength as well as a robust TALE-repressor architecture expands the utility of TALEs for probing mammalian transcription and genome function.

\section{Methods}

Construction of TALE activators, repressors and reporters. All TALE activators and repressors were constructed as previously described using a hierarchical ligation strategy $y^{3,7}$. Briefly, TALE monomers bearing different RVDs were amplified with a common set of primers to add unique ligation adapters ${ }^{3,7}$ to enable ordered assembly of TALE DNA-binding repeats. Arrays of DNA-binding repeats were assembled according to the target TALE-binding sites and cloned into the destination vector ${ }^{3,7}$. The destination vector contains a strong mammalian promoter (EF1 $\alpha$ or cytomegalovirus (CMV)) and the TALE backbone including the cloning sites for inserting customized arrays of TALE DNA-binding domains. The sequences for all constructs used in this study can be found in Supplementary Table S1. To control for differences in the expression of each TALE, all TALEs are in-frame fused with the Gaussia luciferase (Gluc) gene via a 2A linker. The Gluc gene will be translated in an equimolar amount as TALEs. Truncation variants of the KRAB domain, the PIE-1 repression domain (PIE-1), the QA domain within the Ubx gene (Ubx-QA), the IAA28 repression domain (IAA28-RD), Tbx3 repression domain (Tbx3-RD) and the SID were codon optimized for mammalian expression and synthesized with flanking NheI and XbaI restriction sites (Genscript). All repressor domains were cloned into the TALE backbone by replacing 
a

SOX2 TALE

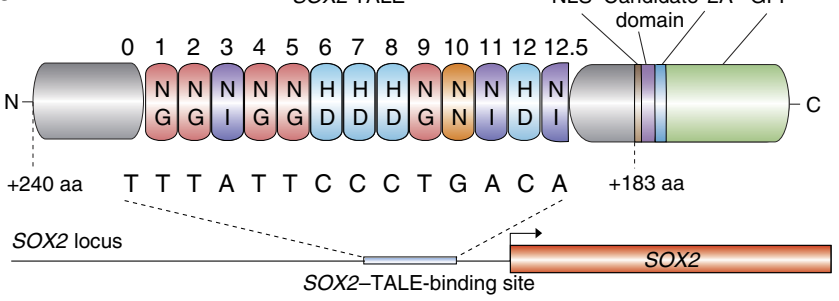

b

\begin{tabular}{cc}
$\begin{array}{c}\text { Candidate } \\
\text { domains }\end{array}$ & $\begin{array}{c}\text { Organismic } \\
\text { origins }\end{array}$ \\
\hline PIE-1 & C. elegans
\end{tabular}

Ubx-QA D. melanogaster

IAA28-RD A. thaliana

SID $\quad$ H. sapiens

Tbx3-RD H. sapiens

KRAB H. sapiens

C

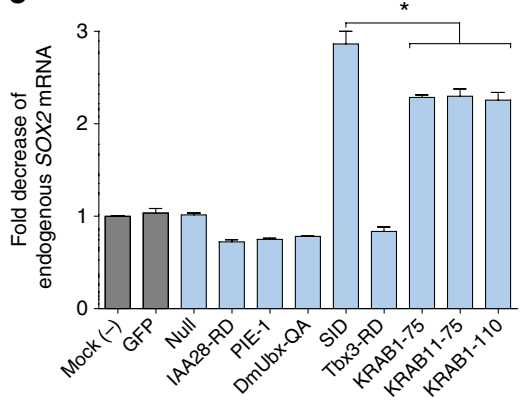

d

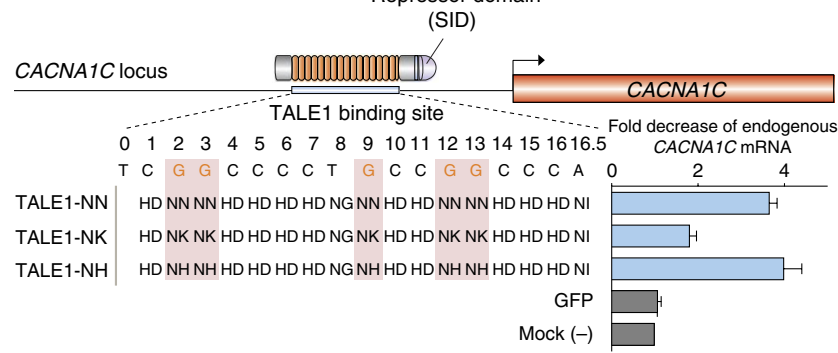

Figure 4 | Development of aTALE transcriptional repressor architecture. (a) Design of SOX2 TALE for TALE-repressor screening. A TALE targeting a 14-bp sequence within the SOX2 locus of the human genome was synthesized as described previously ${ }^{3}$. (b) List of all repressors screened and their host origin (left). Eight different candidate repressor domains were fused to the C-term of the SOX2 TALE. (c) The fold decrease of endogenous SOX2 messenger RNA (mRNA) is measured using qRT-PCR by dividing the SOX2 mRNA levels in mock-transfected cells by SOX2 mRNA levels in cells transfected with each candidate TALE repressor. (d) Transcriptional repression of endogenous CACNA1C. TALEs using NN, NK and NH as the G-targeting RVD were constructed to target a 18-bp target site within the human CACNATC locus (site 1 in Fig. 3). Each TALE is fused to the SID repression domain. NLS, nuclear localization signal; KRAB, Krüppelassociated box. All results are collected from three independent experiments in HEK 293FT cells. Error bars indicate s.e.m.; $n=3$. ${ }^{\star} P<0.05$, Student's $t$-test.

the VP64 activation domain using NheI and XbaI restriction sites. To control for any effect on transcription resulting from TALE binding, we constructed expression vectors carrying the TALE DNA-binding domain alone using PCR cloning. The coding regions of all constructs were completely verified using Sanger sequencing.

All luciferase reporter plasmids were designed and synthesized by inserting the TALE-binding site upstream of the minimal CMV promoter driving the expression of a Cypridina luciferase $(\mathrm{Cluc})$ gene (Fig. 1), similar to minCMVmCherry reporter used in previous studies ${ }^{3}$.

Cell culture and luciferase reporter activation assay. Maintenance of human embryonic kidney cell line HEK 293FT (Invitrogen) were carried out with DMEM supplemented with $10 \%$ fetal bovine serum (HyClone), $2 \mathrm{mM}$ GlutaMAX (Invitrogen), $100 \mathrm{U} \mathrm{ml}^{-1}$ penicillin and $100 \mathrm{\mu g} \mathrm{ml}^{-1}$ streptomycin, under $37^{\circ} \mathrm{C}, 5 \% \mathrm{CO}_{2}$ incubation condition.

Luciferase reporter assays were performed by co-transfecting HEK 293FT cells with TALE-2A-luciferase expression and luciferase reporter plasmids. In the case of the reporter-only control, cells were co-transfected with a control Gaussia luciferase plasmid (pCMV-Gluc, New England BioLabs). HEK 293FT cells were seeded into 24 -well plates the day before transfection at densities of $2 \times 10^{5}$ cells per well. Approximately $24 \mathrm{~h}$ after initial seeding, cells were transfected using Lipofectamine2000 (Invitrogen) following the manufacturer's protocol. For each well of the 24-well plates $700 \mathrm{ng}$ of dTALE and $50 \mathrm{ng}$ of each reporter plasmids were used to transfect HEK 293FT cells.

Dual luciferase reporter assays were carried out with the BioLux Gaussia luciferase flex assay kit and BioLux Cypridina luciferase assay kit (New England Biolabs) following the manufacturer's recommended protocol. Briefly, media from each well of transfected cells were collected $48 \mathrm{~h}$ after transfection. For each sample, $20 \mu \mathrm{l}$ of the media were added into a 96-well assay plate and mixed with each one of the dual luciferase assay mixes. After brief incubation, as indicated in the manufacturer's protocol, luminescence levels of each sample were measured using the Varioskan flash multimode reader (Thermo Scientific).

The activity of each TALE is determined by measuring the level of luciferase reporter induction and calculated as the level of Cluc induction in the presence of TALE activator minus the level of Cluc induction without TALE activator. The activity of each TALE is normalized to the level of TALE expression as determined by the Gluc activity level (each TALE is in-frame fused to 2A-Gluc), to control for differences in cell number, sample preparation, transfection efficiency and protein expression level. The concentration of all DNA used in transfection experiments were determined using gel analysis.

We determined the base preference of each RVD according to the induction of each base-specific reporters by the corresponding RVD screening TALE (RVD-TALE, Fig. 1a). Statistical analyses were performed using one-way analysis of variance (ANOVA) tests. Each RVD was tested by taking the reporter with the highest luciferase activity as the putative preferred base and comparing it with the remaining three bases as a group. For a given RVD, if the putative preferred base gave statistically significant test results $(P<0.05$, one-way ANOVA), we classified that RVD as having a single preferred base, otherwise that RVD is tagged as not having a single preferred base.

Endogenous gene transcriptional activation assay. For the endogenous gene transcriptional level assay to test the biological activities of TALE activators and TALE repressors, HEK 293FT cells were seeded into 24-well plates. $800 \mathrm{ng}$ of TALE plasmid was transfected using Lipofectamine2000 (Invitrogen) according to the manufacturer's protocol. Transfected cells were cultured at $37^{\circ} \mathrm{C}$ for $72 \mathrm{~h}$ before RNA extraction. At least 100,000 cells were harvested and subsequently processed for total RNA extraction using the RNAeasyPlus Mini Kit (Qiagen). Complementary DNA was generated using the High Capacity RNA-to-cDNA Master Mix (Applied Biosystems) according to the manufacturer's recommended protocol. After cDNA synthesis, cDNA from each samples were added to the qRT-PCR assay with the Taqman Fast Advanced PCR Master Mix (Applied Biosystems) using a StepOne Plus qRT-PCR machine. The fold activation in the transcriptional levels of SOX2 and CACNA1C messenger RNA were detected using standard TaqMan Gene Expression Assays with probes having the best coverage (Applied Biosystems; SOX2, Hs01053049_s1; CACNA1C,Hs00167681_m1).

Computational analysis of RVD specificity. To assess the guanine specificity of $\mathrm{NH}$, we performed extensive computational simulations to compare the relative binding affinities between guanine and $\mathrm{NN}$ or $\mathrm{NH}$ using free-energy perturbation (FEP) ${ }^{19,20}$, a widely used approach for calculating binding affinities for a variety of biological interactions, such as ligand-receptor binding, protein-protein interaction and protein-nucleic acid binding ${ }^{21,22}$. We based our calculations on the recently released crystal structure of the TALE PthXo1 bound to DNA (PDB ID: $3 \mathrm{UGM})^{12}$. We used a fragment of the crystal structure containing repeats $11-18$ of PthXo1 (RVD sequence: HD[11]-NG[12]-NI[13]-HD[14]-NG[15]-NN[16]NG[17]-NI[18], the position of each repeat is indicated by the number in square bracket) and the corresponding 18-bp double-stranded DNA molecule containing the TALE-binding sequence $\left(5^{\prime}\right.$-CTACTGTA- $\left.3^{\prime}\right)$ in the centre to compare the binding affinities of RVDs NN, NK and NH for guanine. In order to have a 'smooth' transition from one residue to another, 20 perturbation windows with soft-core potentials have been used. For each mutation, at least five independent runs starting from different initial configurations are performed for better convergence for both the bound (TALE-DNA) and free (TALE-only) states in FEP, with $60+$ ns molecular dynamics simulation time generated each for the underlying conformation space sampling (see ref. 23 for more details). The TALE-DNA complex is then solvated in a $66 \AA \times 77 \AA \times 51 \AA$ water box, with $100 \mathrm{mM} \mathrm{NaCl}$ added to mimic the physiological environment. The total number of atoms in the simulation system is 26,700. The CHARMM22 force field and TIP3P water model were used for the simulation. As the 16th repeat in the structure is NN, we computationally mutated $\mathrm{NN}$ into $\mathrm{NH}$ or $\mathrm{NK}$ and calculated the binding affinity of each configuration (NN:G and NH:G). The affinity was calculated as the gain of free energy $(\Delta \Delta G)$ in the DNA-bound state taking NN:G as reference $(\Delta \Delta G=0)$.

\section{References}

1. Boch, J. et al. Breaking the code of DNA binding specificity of TAL-type III effectors. Science 326, 1509-1512 (2009). 
2. Moscou, M. J. \& Bogdanove, A. J. A simple cipher governs DNA recognition by TAL effectors. Science 326, 1501 (2009).

3. Zhang, F. et al. Efficient construction of sequence-specific TAL effectors for modulating mammalian transcription. Nat. Biotechnol. 29, 149-153 (2011).

4. Morbitzer, R., Romer, P., Boch, J. \& Lahaye, T. Regulation of selected genome loci using de novo-engineered transcription activator-like effector (TALE)-type transcription factors. Proc. Natl Acad. Sci. USA 107, 21617-21622 (2010).

5. Miller, J. C. et al. A TALE nuclease architecture for efficient genome editing. Nat. Biotechnol. 29, 143-148 (2011).

6. Geißler, R. et al. Transcriptional activators of human genes with programmable DNA-specificity. PLoS One 6, e19509 (2011).

7. Sanjana, N. E. et al. A transcription activator-like effector toolbox for genome engineering. Nat. Protoc. 7, 171-192 (2012).

8. Mahfouz, M. M. et al. Targeted transcriptional repression using a chimeric TALE-SRDX repressor protein. Plant Mol. Biol. 78, 311-321 (2012).

9. Bogdanove, A. J. \& Voytas, D. F. TAL effectors: customizable proteins for DNA targeting. Science 333, 1843-1846 (2011).

10. Ayer, D. E., Laherty, C. D., Lawrence, Q. A., Armstrong, A. P. \& Eisenman, R. N. Mad proteins contain a dominant transcription repression domain. Mol. Cell. Biol. 16, 5772-5781 (1996).

11. Huang, P. et al. Heritable gene targeting in zebrafish using customized TALENs. Nat. Biotechnol. 29, 699-700 (2011).

12. Mak, A. N., Bradley, P., Cernadas, R. A., Bogdanove, A. J. \& Stoddard, B. L. The crystal structure of TAL effector PthXo1 bound to its DNA target. Science 335, 716-719 (2012).

13. Batchelder, C. et al. Transcriptional repression by the Caenorhabditis elegans germ-line protein PIE-1. Genes Dev. 13, 202-212 (1999).

14. Tour, E., Hittinger, C. T. \& McGinnis, W. Evolutionarily conserved domains required for activation and repression functions of the Drosophila Hox protein Ultrabithorax. Development 132, 5271-5281 (2005).

15. Tiwari, S. B., Hagen, G. \& Guilfoyle, T. J. Aux/IAA proteins contain a potent transcriptional repression domain. Plant Cell 16, 533-543 (2004).

16. Margolin, J. F. et al. Kruppel-associated boxes are potent transcriptional repression domains. Proc. Natl Acad. Sci. USA 91, 4509-4513 (1994).

17. Mahfouz, M. M. et al. De novo-engineered transcription activator-like effector (TALE) hybrid nuclease with novel DNA binding specificity creates doublestrand breaks. Proc. Natl Acad. Sci. USA 108, 2623-2628 (2011).

18. Scholze, H. \& Boch, J. TAL effectors are remote controls for gene activation. Curr. Opin. Microbiol. 14, 47-53 (2011).

19. Almlof, M., Aqvist, J., Smalas, A. O. \& Brandsdal, B. O. Probing the effect of point mutations at protein-protein interfaces with free energy calculations. Biophys. J. 90, 433-442 (2006).
20. Wang, J., Deng, Y. \& Roux, B. Absolute binding free energy calculations using molecular dynamics simulations with restraining potentials. Biophys. J. 91, 2798-2814 (2006).

21. Zhou, R., Das, P. \& Royyuru, A. K. Single mutation induced H3N2 hemagglutinin antibody neutralization: a free energy perturbation study. J. Phys. Chem. B 112, 15813-15820 (2008).

22. Chodera, J. D. et al. Alchemical free energy methods for drug discovery: progress and challenges. Curr. Opin. Struct. Biol. 21, 150-160 (2011).

23. Xia, Z., Huynh, T., Kang, S. G. \& Zhou, R. Free-energy simulations reveal that both hydrophobic and polar interactions are important for influenza hemagglutinin antibody binding. Biophys. J. 102, 1453-1461 (2012).

\section{Acknowledgements}

We thank the entire Zhang laboratory for their support and advice. L.C. is a Howard Hughes Medical Institute International Student Research fellow. R.Z. is supported by the IBM Blue Gene Science Program. Y.-c.K. and M.C. are supported by MIT Undergraduate Research Opportunities (UROP). F.Z. is supported by a NIH Transformative R01 (1R01NS073124), the McKnight, Gates, Damon Runyon, Kinship, Klingenstein and Simons Foundations, Bob Metcalfe and Mike Boylan. We also thank George Church for helpful comments. Additional sequence information as well as TALE-related protocols can be found at the open source TAL Effector Resources Website (http://www.taleffectors.com).

\section{Author contributions}

L.C. and F.Z. conceived the study and designed all experiments. L.C., Y.-c.K., M.C. and F.Z. performed and analysed all experiments. R.Z. performed the computational analysis of TALE-DNA-binding affinity. L.C., R.Z. and F.Z. wrote the manuscript with support from all authors.

\section{Additional information}

Supplementary Information accompanies this paper at http://www.nature.com/ naturecommunications

Competing financial interests: The authors declare no competing financial interests.

Reprints and permission information is available online at http://npg.nature.com/ reprintsandpermissions/

How to cite this article: Cong, L. et al. Comprehensive interrogation of natural TALE DNA binding modules and transcriptional repressor domains. Nat. Commun. 3:968 doi: $10.1038 /$ ncomms1962 (2012) 